\title{
Pilot Based Channel Estimation in Broadband Power Line Communication Networks
}

\author{
M. Kh. Andari, A. A. Beheshti \\ Electrical Engineering Department, Iran University of Science and Technology, Tehran, Iran \\ Email: m.kh.andari@ieee.org, abeheshti@iust.ac.ir
}

Received February 26, 2012; revised March 25, 2012; accepted April 28, 2012

\begin{abstract}
In this paper pilot based channel estimation is being considered for broadband power line communication (BPLC) networks witch used orthogonal frequency division multiplexing (OFDM) in order to transmit high rate data. To estimate channel in time or frequency some pilot must be used. Number of these pilots and deployment of them is very important for proper estimation in different channel with varying time and frequency. Carrier sense multiple access (CSMA) and hybrid multiple access protocol are taken into consideration in MAC sub-layer. Multilayered perceptions neural network with backpropagation (BP) learning channel estimator algorithm with different pilot deployment compare to classic algorithm in for channel estimating. Simulation results show the proposed neural network estimation decreases bit error rate and therefore network throughput increases.
\end{abstract}

Keywords: BPLC; Back-Propagation Neural Network (BPNN); Channel Estimation; Pilot; Throughput

\section{Introduction}

Driven by global push to enhanced energy management the development of smart grid technology is also being fueled by a powerful combination of utility industry initiative and consumer demand for lower energy costs. With high-speed communications not only we can have smart grid technology for energy management but also we can have a good promising network for numerous indoor communication applications, for example telephone service, home automation and internet access. Although technological progress of access networks to bring broadband services is increasing very fast recently, but high speed data transmission over power lines is still real challenge for communication engineers. Reasonably priced broadband Internet communication to residential customers is now available via cable modems and various flavors of Digital Subscriber Line (DSL). In turn there is a growing need for in-home network to share this single full-time Internet access link while supporting the wide range of digital data and multimedia communication services. The HomePlug AV specification includes both physical and medium access layers and it is completely interoperable with IEEE P1901 Orthogonal frequency division multiplexing (OFDM) draft standard. OFDM was selected as the basic modulation scheme for HomePlug AV (HPAV) due to its ability to provide extreme robust performance in the presence of frequency selective fading. HPAV with slow frequency selective channel is discussed in [1]. In this paper, HomePlug AV with fast frequency selective fading channel is taken to considerations. HPAV has $200 \mathrm{Mbps}$ channel rate to provide a $150 \mathrm{Mbps}$ information rate.

OFDM is a digital multi-carrier modulation scheme, which uses a large number of closely-spaced orthogonal sub-carriers that is particularly suitable for frequency selective channels and high data rates [1,2].

Channel impulse response (CIR) for BPLC channel can be estimated using predetermined pilot symbols in real time in exchange for information rate reduction. In this paper different deployment of pilot based channel estimating are discussed. One of these deployments that send predefined pilot and then send data is discussed in [1]. Here, because of fast frequency selective fading channel, beside these pilots, data inserted pilots deployment are used. Three types of pilot based channel estimations (Least square (LS), minimum mean square error (MMSE), and neural network (NN)) are compared. Our proposed channel estimation is neural network with back-propagation learning method.

The advantage of our method is we do not need knowledge of noise and other statistics of the channel at the expense of complicity in both transmitter and receiver. BPLC channel has various noises. These noises may be different in one channel to others. Because of this reason, having no knowledge of channel parameter our method is the best among these three methods. Disad- 
vantage of neural network is it has more computation for training neural network. In contrast with wireless communication power line communication doesn't have power supply problem. However, more complexity is not a severe problem because it may be removed by powerful processors or Field-programmable gate array (FPGA). Having no problem with power supply and data processing in BPLC networks, bring this benefit that we use more and more adaptive method such as $\mathrm{NN}$ method for estimate BPLC channels everywhere.

In MAC sub-layer, VSMA (hybridized CSMA/CA and TDMA) is used. Relation between PHY bit error rate and MAC layer is discussed. As a result of neural network channel estimation, the BER is decreased and after that throughput and access delay are improved.

The reminder of this paper organized as follow: in Section 2, physical layer and in Section 3 Channel estimation methods are considered. In Section 4 MAC sublayer of BPLC channel is described. Simulation result presented in Section 5, and finally Section 6 contains conclusions. Bold letters are chosen for notation of matrix and vector variables.

\section{Physical Layer}

In physical layer, data rate is $200 \mathrm{Mbps}$. About 3/4 of is rate is information rate, and 1/4 used as channel coding. Scrambler, turbo convolutional coding and random interleaver block, due to harsh channel condition, are used. The Data mapper block accepts integer inputs and produces integer outputs. We can select one of four mapping modes: Binary to Gray, Gray to Binary. For example, with binary to gray mapping vector $[0,1,2,3,4,5,6,7]$ is mapped to $[0,13,2,6,7,5,4]$.

Cyclic prefix is often used in conjunction with OFDM modulation in order to retain sinusoids' properties in multipath channels.

Peak limiter used for limiting OFDM signal's peak. Analog front end (AFE) is the analog portion of a circuit which precedes $\mathrm{A} / \mathrm{D}$ conversion. In receiver all of operations described above are done inversely. AVG block is automatic gain control. Figure 1 shows transmitter and receiver used for physical layer. Further detail about transmitter and receiver are finding in [1].

\subsection{OFDM Symbol's Pilot Deployment}

Different possibilities exist for allocating pilots in the time-frequency domain of an OFDM system. We discuss three such possibilities as illustrated in Figure 2. An entire OFDM symbol may be allocated as pilot as shown in Figure 2(a). Such an allocation will be beneficial for channel estimation in fast frequency selective fading channels at the expense of sacrificing data rate.

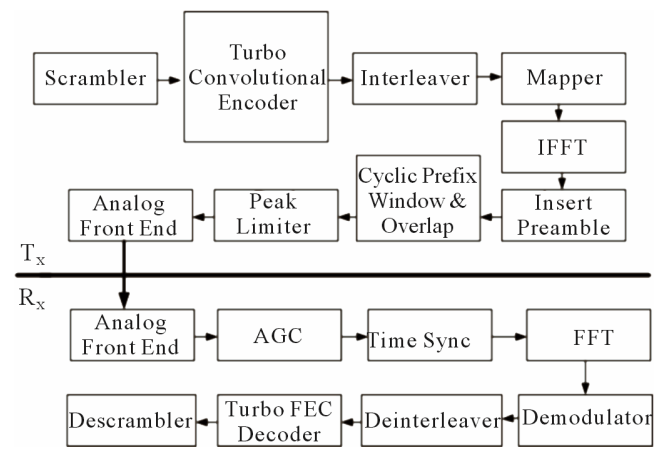

Figure 1. Physical layer transmitter and receiver.

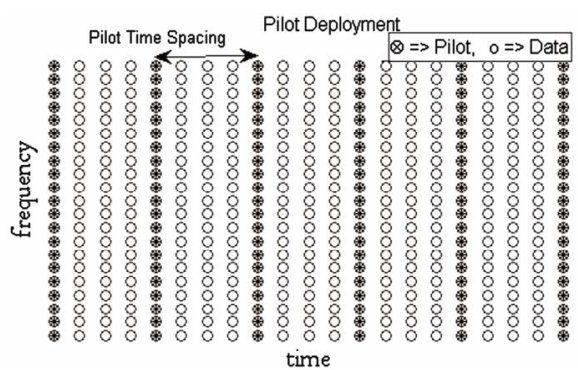

(a)

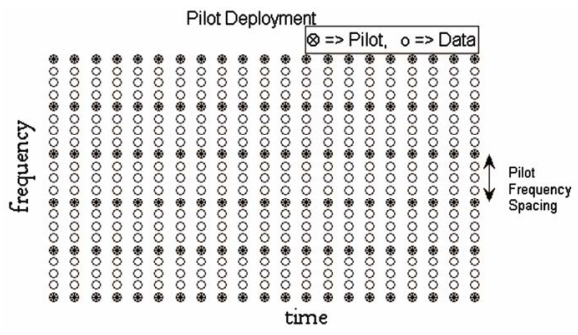

(b)

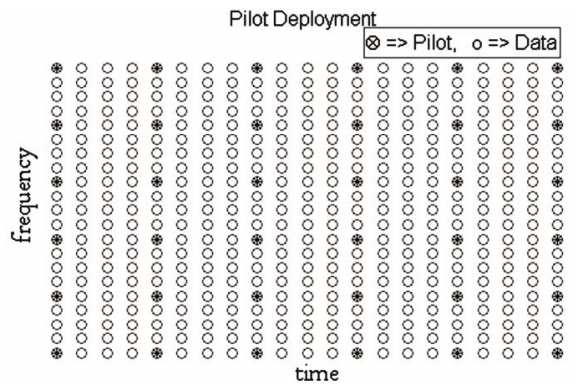

(c)

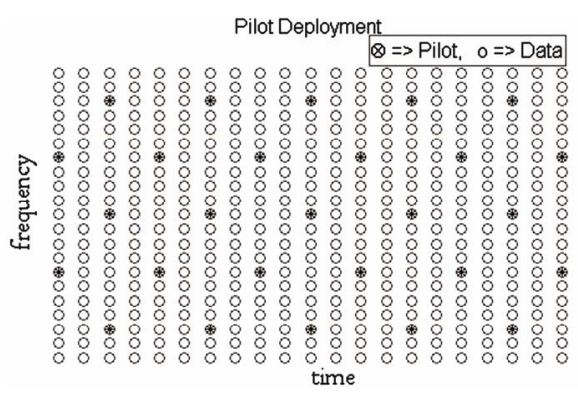

(d)

Figure 2. Different deployment of inserted pilot. 
Pilots may be transmitted on individual sub-carriers during the entire transmission period as shown in Figure 2(b). Such a strategy will be advantageous in fast time varying channels.

Pilots may be allocated in spaced intervals in time and frequency as illustrated in Figure 2(c) and Figure 2(d). Depending upon the time-frequency pilot spacing and channel properties.

Channel estimation in OFDM is a two-dimensional problem i.e., channel needs to be estimated in time-frequency domain. Hence two-dimensional methods could be applied to estimate the channel from pilots. However, due to the computational complexity of two-dimensional estimators, the scope of channel estimators can be limited to one dimensional. Two or one dimensional channel estimation methods can be characterized into three types:

- Simple linear interpolation.

- Orthogonal polynomials interpolation.

- Wiener filtering using second-order statistics of the channel.

The focus of this paper has been on one dimensional and fast frequency selective channel for fast fading channel and non-inserted pilot between data for slow fading channel.

\subsection{Channel and Noise Models}

Indoor power line channel has frequency and time variant behavior. The power line link may be subjected to various constraints such as attenuation because of interference, multipath over the electrical wiring, or electrical wiring crosstalk effects. Then, we can describe this channel like this (1).

$$
h(\tau ; t)=\sum_{p=0}^{N_{p}-1} \alpha_{p}(t) \delta\left(\tau-\tau_{p}(t)\right)
$$

where $\alpha_{p}$ is $p^{\text {th }}$ fading channel coefficient, $p$ is path number, $N_{p}$ is number of multipath, $\tau_{p}$ is $p^{\text {th }}$ delay path. Fading channel coefficients are independent and is a Rician distributed random variable. Path delays are independent Gaussian random variables [1,3].

Additive white Gaussian noise is not the dominant noise in power line channels. The different types of noise that can be identified on and around the wiring are:

- Impulse noise from stops and starts of electrical devices.

- Broadband white noise whose power spectral density is the same for all frequencies (background noise).

- Periodic noise at multiple frequencies.

- Harmonic noise composed of multiple frequencies used by electrical equipment connected to the network and which are multiples of the line frequency (for example, $50 \mathrm{~Hz}$ yields harmonics of $300 \mathrm{~Hz}, 600$ $\mathrm{Hz}$, and so forth).

Overall, the noise is quantified by the signal-to-noise ratio, or SNR, generally measured in decibels $(\mathrm{dB})$.

Model of the background noise is Nakagami- $m$ that its PDF can be written as [4]

$$
p(r)=\frac{2}{\Gamma(m)}\left(\frac{m}{\Omega}\right)^{m} r^{2 m-1} e^{-\frac{m r^{2}}{\Omega}}
$$

wher $r$ is a random variable, $\Gamma(\cdot)$ is the Gamma function, $p$ is the probability of the corresponding random variable, $m$ is defined as the ratio of moments, and $\Omega$ is the mean power of the random variable [1]. Figure 3 shows noise and coefficients of sub-channels.

\section{Channel Estimation}

In BPL system OFDM modulation is used. While evaluating OFDM system, we may assume perfect knowledge of the channel for equalization to find the upper limit of OFDM system performance; such perfect channel knowledge is not available in real-life and needs to be estimated. Channel estimation can be done in various ways: with or without the help of a parametric model, with the use of frequency and/or time correlation properties of the PBL channel, blind or pilot (training) based, adaptive or not. Non-parametric methods attempt to estimate the quantities of interest (for example the frequency response) without relying on a specific channel model. Conversely, parametric estimation assumes a definite channel model, determines the parameters of this model and infers the quantities of interest.

Spaced-time and spaced-frequency correlations are specific properties of channel that can be incorporated in the estimation method, improving the quality of estimate. Pilot based estimation methods are the most commonly used methods which are applicable in BPL systems where the sender emits some known signal. Blind estimation, on the other hand, relies on some properties of the signal (e.g., cylco-stationarity of the signal) and is rarely used in practical OFDM systems. Adaptive channel estimation methods are typically used for rapidly time-varying channel.

Different methods can then be applied to estimate the channel over all sub-carrier frequencies and not just at

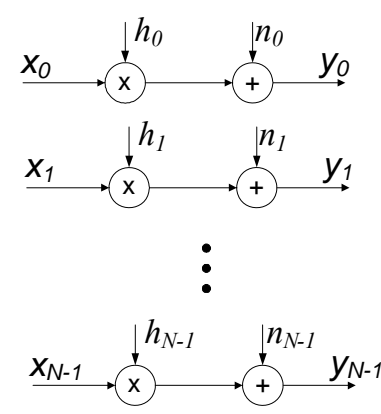

Figure 3. Noise and coefficients of sub-channels. 
pilot sub-carrier frequencies.

Here, we derive three estimator based on physical layer and system model in previous section. Assume $X$ is Fourier transforming of the transmitted data. Then $y$, the received vector in frequency domain, is:

$$
y=X h+n
$$

or

$$
\left[\begin{array}{c}
y^{(1)} \\
\vdots \\
y^{(k)}
\end{array}\right]=\left[\begin{array}{lll}
X^{(1)} & & \\
& \ddots & \\
& & X^{(k)}
\end{array}\right]\left[\begin{array}{c}
h^{(1)} \\
\vdots \\
h^{(k)}
\end{array}\right]+\left[\begin{array}{c}
n^{(1)} \\
\vdots \\
n^{(k)}
\end{array}\right]
$$

where $h$ is frequency response of BPLC channel and $n$ is Fourier transform of additive BPLC channel noise. OFDM modulation is used to transmit data. $k$ is number of sub-channels in the OFDM block. Since BPL channel is often a multipath fading channel, inter-symbol interference (ISI) in the received signal is existed. To remove ISI, various types of equalizers can be used before detection. Detection algorithms which based on trellis search (such as MLSE or MAP) have a good performance, but even now have too much computation. These detectors need information on channel impulse response (CIR) that can be provided by a separate channel estimator. Figure 4 shows block diagram for a system utilizing channel estimator in each subcarrier $[5,6]$.

\section{LS, MMSE and NN Estimation}

Least square channel estimation for estimating coefficients of channel frequency response for sub-channels calculate like this

$$
\hat{h}_{l s}=X^{-1} y
$$

This solution exists and is unique, provided that the inverse of $X^{T} X$ exists. And minimum mean square error channel estimating are gained these coefficient like this

$$
\hat{h}_{\text {mmse }}=R_{h h}\left(R_{h h}+\sigma^{2}\left(X X^{-1}\right)^{H}\right)^{-1} \hat{h}_{l s}
$$

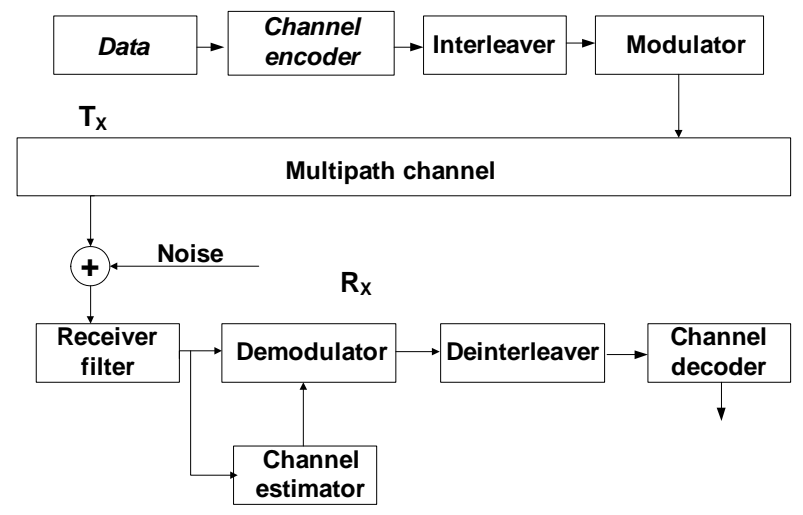

Figure 4. Block diagram for channel estimator. where $\sigma^{2}$ is variance of noise and $R_{h h}$ is auto-covariance matrix of channel coefficients [5].

To gain CIR, MLP neural network with backpropagation learning algorithm are used. Because of neural network nature, OFDM symbol which is a complex signal must be separated into real and imaginary parts. Neural network have two inputs and two outputs. Two outputs of neural network are real and imaginary part of CIR in the frequency domain. MLP structure for neural network channel estimation is shown in Figure 5. In this structure $f$ is sigmiod function, $b_{i}$ is bias, $X_{i}$ is input data, $O_{j}$ is output of hidden layer, $O_{k}$ is output of system, $W_{i j}$ is input-to-hidden layer weights and $W_{j k}$ is hidden-to-output layer weights of neural network structure [7].

\section{MAC Sub-Layer}

MAC sub-layer offers both connection oriented and connectionless communication between source and destination station. In connection-oriented, time division multiple access (TDMA) has major rule and in the other one, carrier sense multiple access collision avoidance (CSMA/CA) has vital rule. VSMA algorithm is hybridized CSMA/CA with TDMA to guarantee latency and jitter requirement for real-time communication. For quality of service (QoS), in CSMA, different priority is needed. In this paper, 4 priorities are taken, and for better QoS these priorities have 2 access categories (AC). Then we have 8 ACs. The stations with higher priority can transmit their frame with more probability. Exponential backoff algorithm is done among station with the same priority. According to backoff algorithm, each station waits a random time after priority resolution (PR) then transmits its frame. Contention window $(\mathrm{CW})$ size for a station is maximum time that station waits to transmit its frame. The sizes of CWs are defined through Table $\mathbf{1}$ as function of ACs. In Figure 6 timing of data transmission is shown. In this figure CIFS and RISF stand for contention inter frame spacing and response inter frame spacing, respectively. In $[8,9]$ fixed and dynamic CW for VSMA algorithm is discussed $[10,11]$. One can find details about VSMA in these two references. In this paper, influence of bit error rate in PHY layer is discussed. If BER in PHY layer is low then when a station access the medium can transmit frame without more error. With coded data, low error occurred in frame can be corrected and acknowledgement frame in RIFS can transmit to transmitter with high probability. Flow chart in Figure $\mathbf{7}$ shows VSMA procedure in details.

\section{Simulation Results}

Three channel estimations (LS, MMSE, and NN) are compared together in slow frequency selective channel. In Figure 8, the simulation results of these channel esti- 
Table 1. CW selection mechanism based on priority of service [6].

\begin{tabular}{|c|c|c|c|}
\hline AC & Collision & Overhearing collision & Success \\
\hline 1 & $\min \left(C W+\frac{l_{c}}{2}, C W_{\text {max }}\right)$ & $\min \left(C W+l_{c}, C W_{\max }\right)$ & $\max \left(\frac{C W}{2}, C W_{\min }\right)$ \\
\hline 2 & $\begin{array}{l}\min \left(C W+\frac{l_{c}}{2}, C W_{T h}\right) \text { if } C W<C W_{T h} \\
\max \left(2 C W, C W_{\max }\right) \text { if } C W \geq C W_{T h}\end{array}$ & $\begin{array}{l}\min \left(C W+l_{c}, C W_{T h}\right) \text { if } C W<C W_{T h} \\
\min \left(\frac{C W}{2}, C W_{\max }\right) \text { if } C W \geq C W_{T h}\end{array}$ & $\max \left(\frac{C W}{4}, C W_{\min }\right)$ \\
\hline 3 & $\min \left(2 C W, C W_{\max }\right)$ & $\min \left(4 C W, C W_{\max }\right)$ & $\begin{array}{r}\max \left(\frac{C W}{2}, C W_{T h}\right) C \text { if } W \geq C W_{T h} \\
\max \left(C W-l_{s}, C W_{\min }\right) \text { if } C W<C W_{T h}\end{array}$ \\
\hline 4 & $\min \left(2.5 C W, C W_{\max }\right)$ & $\min \left(5 C W, C W_{\max }\right)$ & $\max \left(C W-l_{s}, C W_{\min }\right)$ \\
\hline
\end{tabular}

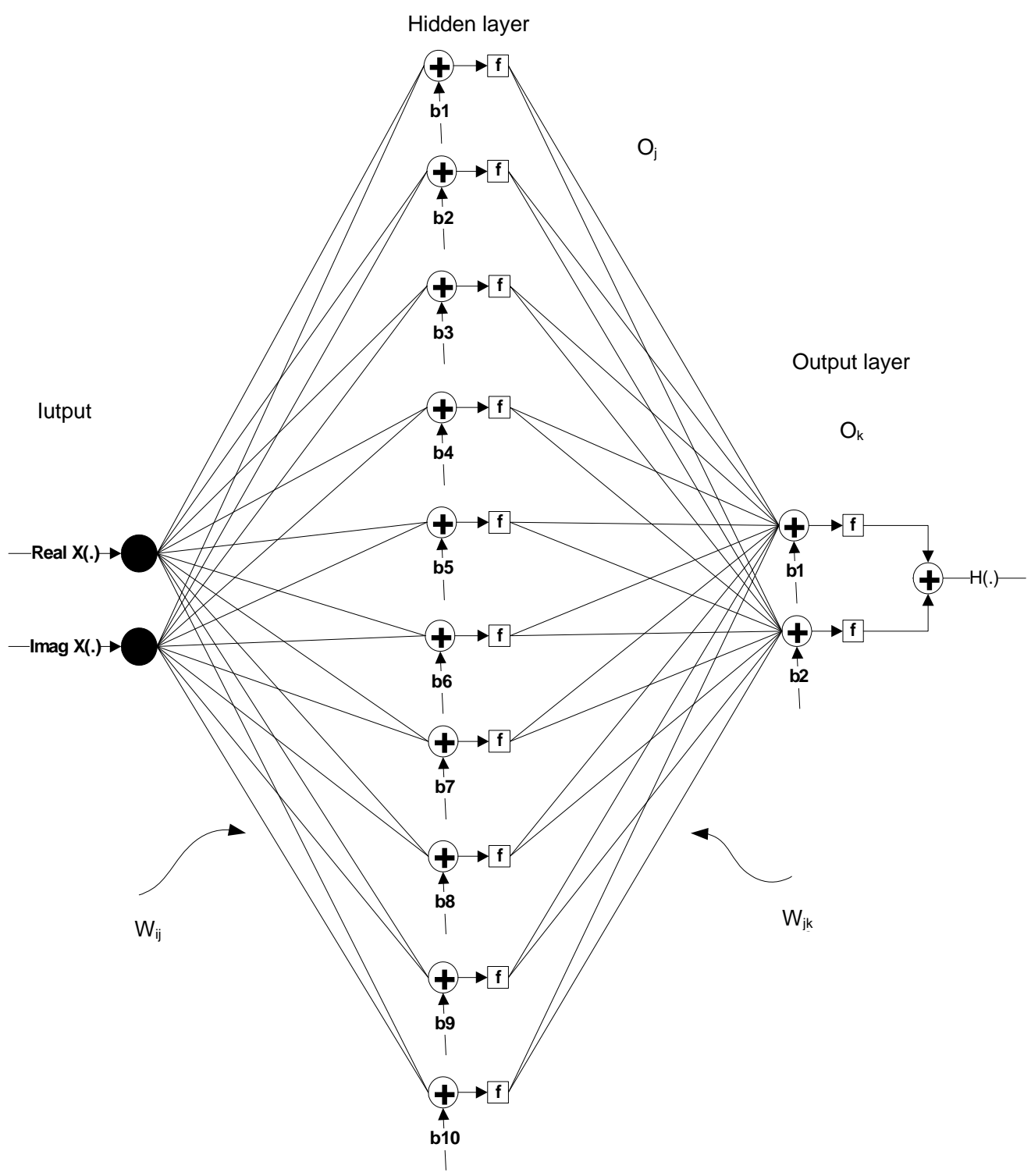

Figure 5. MLP structure for channel estimation. 


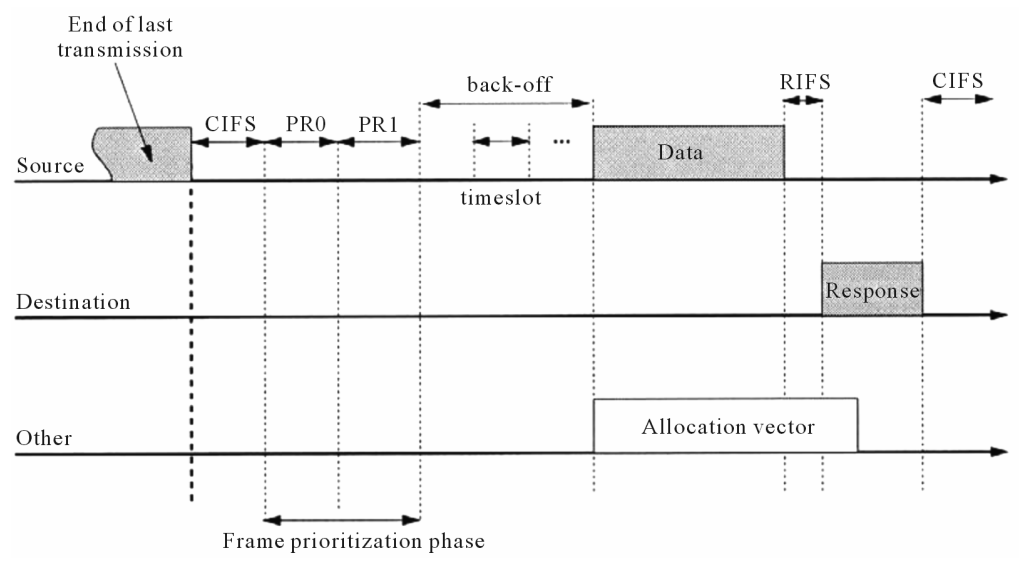

Figure 6. Timing of data transmission for a transmitter and a receiver.

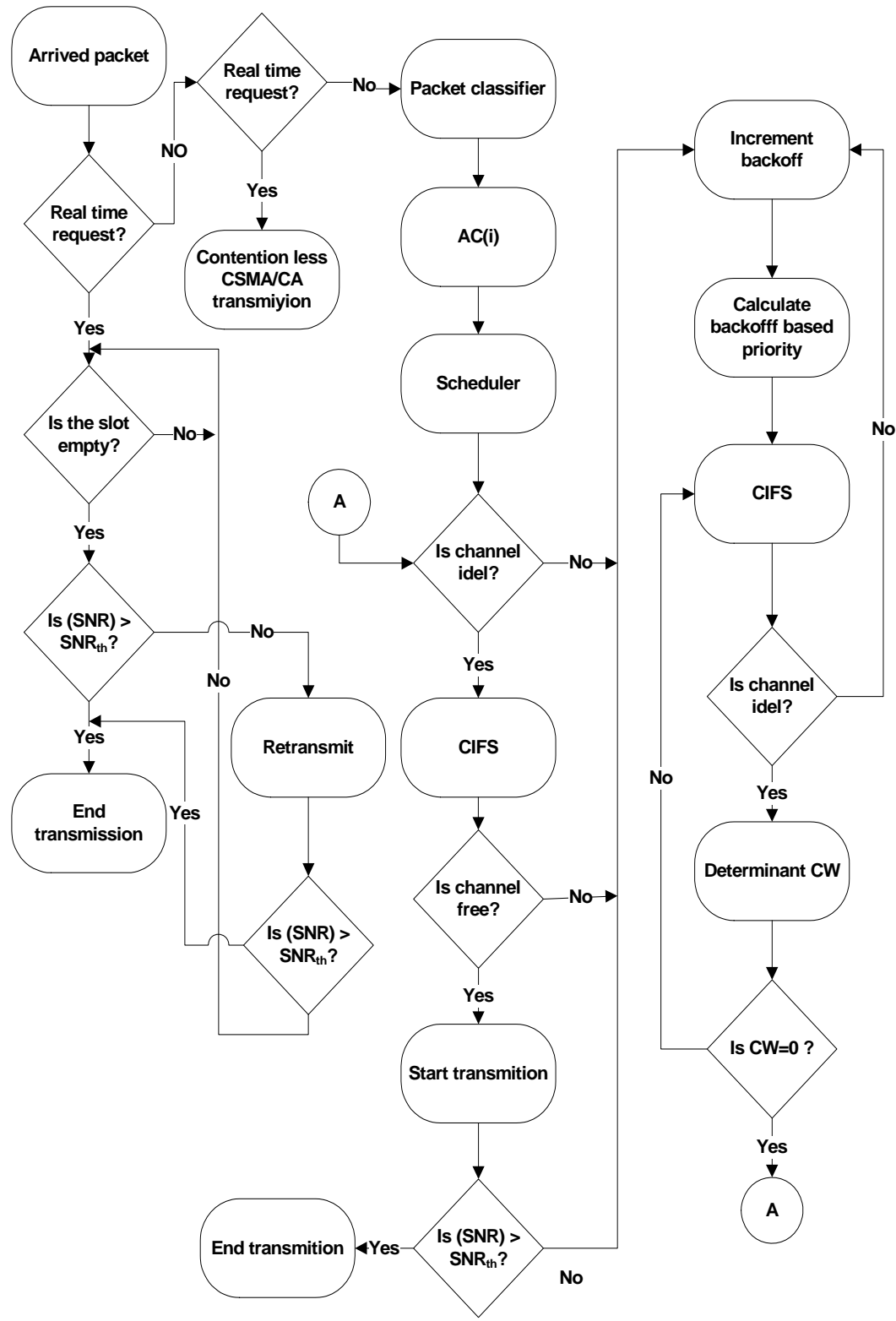

Figure 7. Flowchart of MAC scheme [6]. 


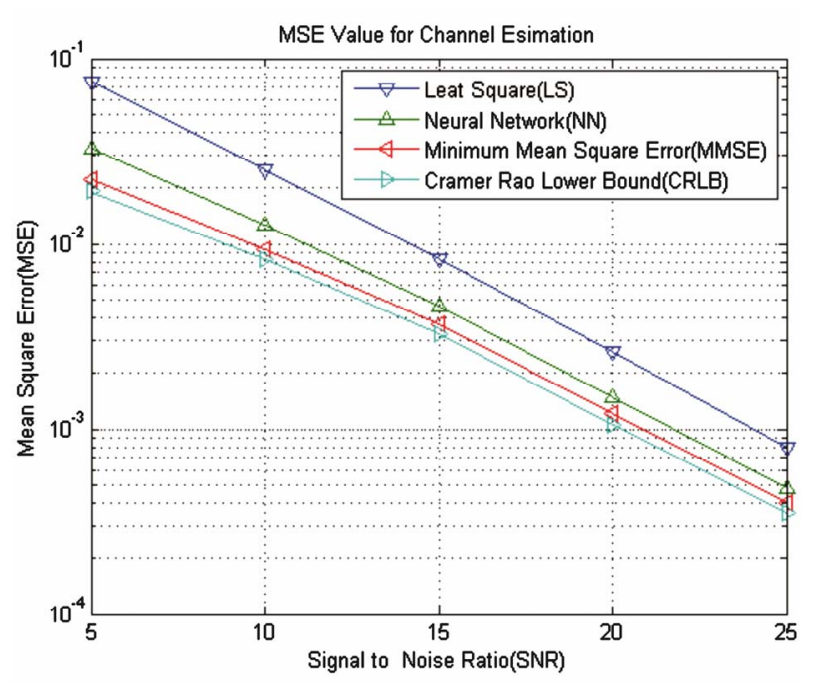

Figure 8. MSE vs. SNR (dB) in non-pilot inserted slow frequency selective fading channel.

mation are shown. In these figure mean square error of estimations have plotted as function of signal to noise ratio. To compare MSE simulation result with theoretical value, Cramer-Rao lower bound (CRLB) is plotted. Mean square error between channel coefficient $h$ and estimated channel coefficient $h$ is gained with simulation. This Figure shows that performance of MMSE is better than LS and neural network (NN) estimation, and $\mathrm{NN}$ performance is better than LS estimation. In simulation, for all of estimators, channel estimations are done in frequency domain. Background noise is additive Gaussian noise. Without loss of generality, for simplification of simulation, we can suppose in only impulsive noise and AWGN noise are existed in power line. This is because of the fact that the other noise can be removed by powerful coding such as turbo coding. Impulsive noise can be made by another AWGN noise which is added to above noise. The power of this noise is 4 to 5 times greater than background noise with duration of about 2 milliseconds.

The modulation is BPSK and FFT size is 64 points. MLP network has one hidden layer with 10 neurons. In hidden layer sigmiod activation function and in output layer linear activation function is used. We have used Monte-Carlo simulation to generate $R_{h h}$ for MMSE estimation. For drawing Figure 8, simulation is repeated fifteen times and mean of the results are calculated. Figure 9 compares the channel coefficient mean square error of fast frequency selective fading channel for pilot based and non-pilot based method. Table 2 shows the other details about simulation.

In Figure 10 and Figure 11, show throughput as function of number of station and packet size for slow and fast frequency selective fading channel, respectively in $\mathrm{SNR}=10 \mathrm{~dB}$.

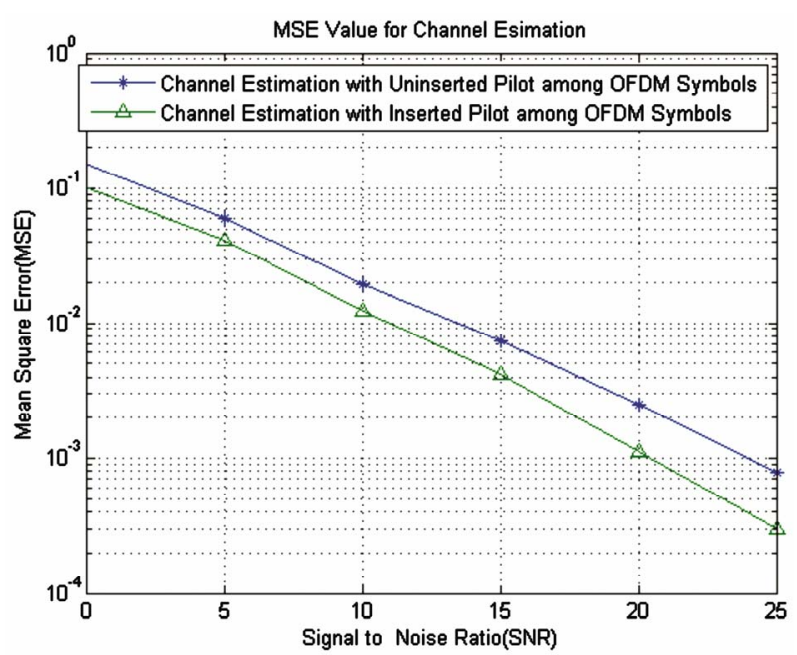

Figure 9. MSE vs. SNR (dB) in pilot inserted and non-pilot inserted channel estimation in fast frequency selective fading channel.

Table 2. Time slot values and other parameters used for simulation.

\begin{tabular}{cc}
\hline Protocol & VSMA \\
\hline Physical Channel Rate & $200 \mathrm{Mbps}$ \\
CIFS & $38.84 \mu \mathrm{sec}$ \\
PRS0, PRS1 & $38.84 \mu \mathrm{sec}$ \\
Idle Slot Time & $38.84 \mu \mathrm{sec}$ \\
RIFS & $38.84 \mu \mathrm{sec}$ \\
Mac Frame Size & $100-2000$ Byte \\
\hline
\end{tabular}

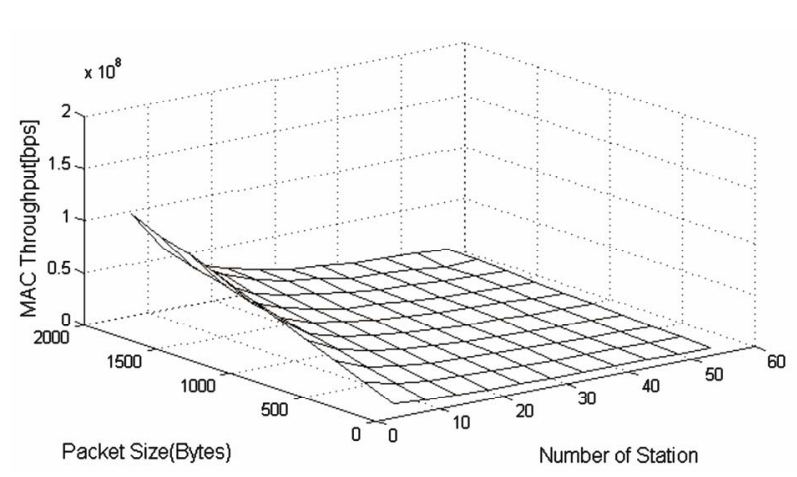

Figure 10. Throughput in VSMA with dynamic CW in pilot inserted in slow frequency selective fading channel.

\section{Conclusion}

In this paper, fast and slow fading of BPL channel are discussed. We have introduced pilot based channel estimation with different deployment. One of these deployment is proper in harsh condition of BPL channel that have better performance in fast fading and frequency selective channel. The effect of BER in MAC layer is 


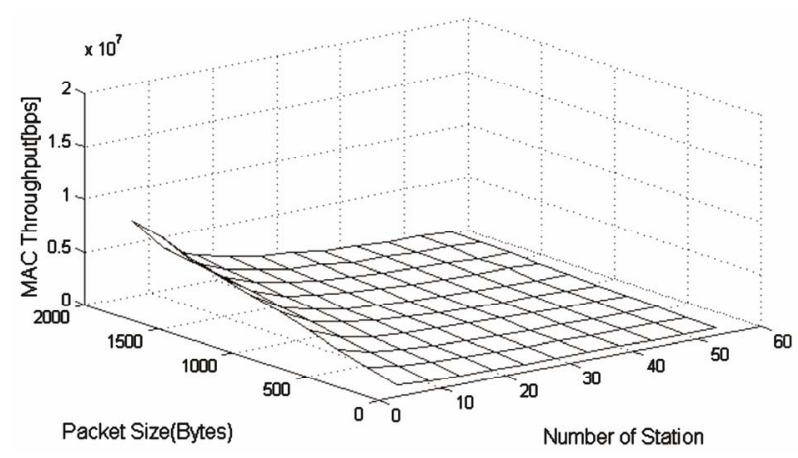

Figure 11. Throughput in VSMA with dynamic CW in pilot inserted in fast frequency selective fading channel.

analyzed. For future work, one may estimate channel in both time and frequency domain then interpolate channel coefficient in both, time and frequency, dimention.

\section{Acknowledgements}

Thanks to Iran Telecommunication Research Center that undertook the moral and financial support of this research.

\section{REFERENCES}

[1] M. K. Andari and S. A. A. Beheshti, "MAC Sub-Layer Analysis with Channel Estimation in Broadband Power Line Communication," Communication and Network Journal, Vol. 3, No. 3, 2011, pp. 141-148.

[2] O. Edfors, et al., "An Introduction to Orthogonal Frequency-Division Multiplexing," Research Report, Luleå University of Technology, Sweden, 1996.

[3] Y.-H. Kim, S.-C. Kim and H. Myong Oh, "OFDM Receiver Performance Analysis with Measured Power Line Channel Model for Coded OFDM System, Power Line Communications and Its Applications," 2005 Interna- tional Symposium on Digital Object Identifier, Vancouver, 23-26 May 2005, pp. 201-205.

[4] X. Carcelle, "Power Line Communications in Practice," Artech House, Boston, 2009, pp. 19-20.

[5] L. L. Scharf, "Statistical Signal Processing: Detection, Estimation, and Time Series Analysis," University of Colorado Boulder, Boulder, 1991, pp. 325-327.

[6] M. Debbah, "Short Introduction to OFDM," White Paper, Mobile Communications Group, Institut Eurecom, 2004.

[7] T. Necmi and S. M. Nuri, "Back Propagation Neural Network Approach for Channel Estimation in OFDM System, Wireless Communications, Networking and Information Security (WCNIS)," 2010 IEEE International Conference, Anchorage, 3-8 May 2010, pp. 265-268.

[8] R. Nakkeeran and A. Rajesh, "Performance Analysis of Powerline Communication for Local Area Networking," International Conference on Control, Automation, Communication and Energy Conservation, Tamilnadu, 4-6 June 2009, pp. 1-5.

[9] A. Rajesh, M. Rathinasabapathy and R. Nakkeeran, "Performance Analysis of Hybrid Protocol Based on Dynamic Contention Window for Power Line Communication Systems," First Asian Himalayas International Conference on Internet, Kathmandu, 3 November 2009, pp. 1-7.

[10] R. Nakkeeran and A. Rajesh, "Performance Analysis of Powerline Communication for Local Area Networking," International Conference on Control, Automation, Communication and Energy Conservation, Tamilnadu, 4-6 June 2009, pp. 1-5.

[11] A. Rajesh, M. Rathinasabapathy and R. Nakkeeran, "Performance Analysis of Hybrid Protocol Based on Dynamic Contention Window for Power Line Communication Systems," First Asian Himalayas International Conference on Internet, Kathmandu, 3 November 2009. doi:10.1109/AHICI.2009.5340267 